\title{
Functional Model of Dissipative Fourth Order Differential Operators
}

\author{
Hüseyin TUNA
}

\begin{abstract}
In this paper, maximal dissipative fourth order operators with equal deficiency indices are investigated. We construct a self adjoint dilation of such operators. We also construct a functional model of the maximal dissipative operator which based on the method of Pavlov and define its characteristic function. We prove theorems on the completeness of the system of eigenvalues and eigenvectors of the maximal dissipative fourth order operators.
\end{abstract}

\section{Introduction}

The spectral analysis of non-selfadjoint operators is based on ideas of the functional model and dilation theory rather than on traditional resolvent analysis and Riesz integrals. Using a functional model of a non-selfadjoint operator as a principal tool, spectral properties of such operators are investigated. The functional model of non-selfadjoint dissipative operators plays an important role within both the abstract operator theory and its more specialized applications in other disciplines. The construction of functional models for dissipative operators, natural analogues of spectral decompositions for selfadjoint operators is based on Sz. Nagy-Foias dilation theory [19] and Lax-Phillips scattering theory [18]. Pavlov's approach ([21-23]) to the model construction of dissipative extensions of symmetric operators was followed by Allahverdiev in his works

Key Words: Dilation, Dissipative operator, Completeness of the system of eigenvectors and associated vectors, Scattering matrix, Functional model, Characteristic function.

2010 Mathematics Subject Classification: 47A20, 47A40.

Received: March, 2012.

Revised: June, 2012.

Accepted: July, 2012. 
[1-5] and some others, and by the group of authors [6-8], where the theory of the dissipative Schrödinger operator on a finite interval was applied to the problems arising in the semiconductor physics. In [9-12], Pavlov's functional model was extended to (general) dissipative operators which are finite dimensional extensions of a symmetric operator, and the corresponding dissipative and Lax-Phillips scattering problems were investigated in some detail.

The organization of this document is as follows: In Section 2, we construct a space of boundary values of the minimal operator and describe all maximal dissipative, maximal accretive, selfadjoint and other extensions of dissipative fourth order differential operators in terms of boundary conditions. Furthermore, we construct a selfadjoint dilation of the dissipative fourth order differential operator. In Section 3, we present its incoming and outgoing spectral representations which makes it possible to determine the scattering matrix of the dilation according to the Lax and Phillips scheme $[17,18]$. Later, a functional model of the dissipative fourth order differential operator is constructed by methods of Pavlov [21-23] and define its characteristic functions. Finally, in Section 4, we proved a theorem on completeness of the system of eigenvectors and associated vectors of dissipative operators under consideration. In the present paper, we extend the results of [1-5] to the more general eigenvalue problem for fourth order differential operators.

\section{Selfadjoint Dilation of Dissipative Fourth Order Dif- ferential Operators}

We will consider the differential expression

$$
l(y)=y^{(4)}+q(x) y, \quad 0 \leq x<+\infty
$$

where $q(x)$ is a real continuous function in $[0, \infty)$.

Let $L_{0}$ denote the closure of the minimal operator generated by $(2.1)$ and by $D_{0}$ its domain. Besides, we denote by the set of all functions $y(x)$ from $L_{2}(0, \infty)$ whose first three derivatives are locally absolutely continuous in $[0, \infty)$ and $l(y) \in L_{2}(0, \infty) ; D$ is the domain of the maximal operator $L$. Furthermore $L=L_{0}^{*}[20]$.

Suppose that $q(x)$ be a function such that the operator $L_{0}$ has defect index $(4,4)$. Let $v_{1}(x), v_{2}(x), v_{3}(x)$ and $v_{4}(x)$ be four linearly independent solutions of the equation $l(y)=0$ satisfying the conditions at $x=0$ :

$$
\begin{aligned}
& v_{1}(0)=1, v_{1}^{\prime}(0)=0, v_{1}^{\prime \prime}(0)=0, v_{1}^{\prime \prime \prime}(0)=0, \\
& v_{2}(0)=0, v_{2}^{\prime}(0)=1, v_{2}^{\prime \prime}(0)=0, v_{2}^{\prime \prime \prime}(0)=0, \\
& v_{3}(0)=0, v_{3}^{\prime}(0)=0, v_{3}^{\prime \prime}(0)=1, v_{3}^{\prime \prime \prime}(0)=0, \\
& v_{4}(0)=0, v_{4}^{\prime}(0)=0, v_{4}^{\prime \prime}(0)=0, v_{4}^{\prime \prime \prime}(0)=1,
\end{aligned}
$$


and their Wronskian equals to one. Since $L_{0}$ has defect index $(4,4)$, then $v_{1}, v_{2}, v_{3}, v_{4} \in L_{2}(0, \infty)$.

Let's define by $\Gamma_{1}, \Gamma_{2}$ the linear maps from $D$ to $\mathbb{C}^{4}$ by the formula

$$
\Gamma_{1} f=\left(\begin{array}{c}
f(0) \\
f^{\prime}(0) \\
{\left[f, v_{2}\right]_{\infty}} \\
{\left[f, v_{1}\right]_{\infty}}
\end{array}\right), \Gamma_{2} f=\left(\begin{array}{c}
f^{\prime \prime \prime}(0) \\
f^{\prime \prime}(0) \\
{\left[f, v_{4}\right]_{\infty}} \\
{\left[f, v_{3}\right]_{\infty}}
\end{array}\right)
$$

where

$[y, z]_{x}=\left[y^{\prime \prime \prime}(x) z(x)-y(x) z^{\prime \prime \prime}(x)\right]-\left[y^{\prime \prime}(x) z^{\prime}(x)-y^{\prime}(x) z^{\prime \prime}(x)\right],(0 \leq x<\infty)$

Lemma 1. For arbitrary $y, z \in D$

$$
(L y, z)_{L^{2}}-(y, L z)_{L^{2}}=\left(\Gamma_{1} y, \Gamma_{2} z\right)_{\mathbb{C}^{4}}-\left(\Gamma_{2} y, \Gamma_{1} z\right)_{\mathbb{C}^{4}} .
$$

Proof. We know that every $f, g \in D$

$$
[f, g](x)=\left|\begin{array}{cc}
{\left[v_{2}, g\right]_{x}} & {\left[g, v_{4}\right]_{x}} \\
{\left[v_{2}, f\right]_{x}} & {\left[f, v_{4}\right]_{x}}
\end{array}\right|+\left|\begin{array}{ll}
{\left[v_{1}, g\right]_{x}} & {\left[g, v_{3}\right]_{x}} \\
{\left[v_{1}, f\right]_{x}} & {\left[f, v_{3}\right]_{x}}
\end{array}\right|
$$

(see [13]). For every $y, z \in D$, we have Green's formula

$$
(L y, z)_{L^{2}}-(y, L z)_{L^{2}}=[y, \bar{z}]_{\infty}-[y, \bar{z}]_{0} .
$$

Then

$$
\begin{aligned}
\left(\Gamma_{1} y, \Gamma_{2} z\right)_{\mathbb{C}^{4}}-\left(\Gamma_{2} y, \Gamma_{1} z\right)_{\mathbb{C}^{4}}= & y(0) \bar{z}^{\prime \prime \prime}(0)-\bar{z}(0) y^{\prime \prime \prime}(0) \\
& +y^{\prime \prime}(0) \bar{z}^{\prime}(0)-\bar{z}^{\prime \prime}(0) y^{\prime}(0) \\
& +\left[y, v_{2}\right]_{\infty}\left[\bar{z}, v_{4}\right]_{\infty}-\left[\bar{z}, v_{2}\right]_{\infty}\left[y, v_{4}\right]_{\infty} \\
& +\left[y, v_{1}\right]_{\infty}\left[\bar{z}, v_{3}\right]_{\infty}-\left[\bar{z}, v_{1}\right]_{\infty}\left[y, v_{3}\right]_{\infty} .
\end{aligned}
$$

From the conditions (2.3), we have

$$
\left(\Gamma_{1} y, \Gamma_{2} z\right)_{\mathbb{C}^{4}}-\left(\Gamma_{2} y, \Gamma_{1} z\right)_{\mathbb{C}^{4}}=[y, \bar{z}]_{\infty}-[y, \bar{z}]_{0}
$$

Hence

$$
(L y, z)_{L^{2}}-(y, L z)_{L^{2}}=\left(\Gamma_{1} y, \Gamma_{2} z\right)_{\mathbb{C}^{4}}-\left(\Gamma_{2} y, \Gamma_{1} z\right)_{\mathbb{C}^{4}}
$$

Lemma 2. For any complex numbers $\alpha_{0}, \alpha_{1}, \alpha_{2}, \alpha_{3}, \beta_{0}, \beta_{1}, \beta_{2}$ and $\beta_{3}$, there is a function $y \in D$ satisfying

$$
\begin{gathered}
y(0)=\alpha_{0}, y^{\prime}(0)=\alpha_{1}, y^{\prime \prime}(0)=\alpha_{2}, y^{\prime \prime \prime}(0)=\alpha_{3} \\
{\left[y, v_{1}\right]_{\infty}=\beta_{0},\left[y, v_{2}\right]_{\infty}=\beta_{1},\left[y, v_{3}\right]_{\infty}=\beta_{2},\left[y, v_{4}\right]_{\infty}=\beta_{3}}
\end{gathered}
$$


Proof. Let $f$ be an arbitrary element of $L_{2}(0, \infty)$ satisfying followings:

$$
\begin{aligned}
& \left(f, v_{1}\right)_{L^{2}}=\beta_{0}-\alpha_{3},\left(f, v_{3}\right)_{L^{2}}=\beta_{2}-\alpha_{1}, \\
& \left(f, v_{2}\right)_{L^{2}}=\beta_{1}+\alpha_{2},\left(f, v_{4}\right)_{L^{2}}=\beta_{3}+\alpha_{0} .
\end{aligned}
$$

There is such a $f$, even among the linear combinations of $v_{1}, v_{2}, v_{3}$ and $v_{4}$. If we set $f=c_{1} v_{1}+c_{2} v_{2}+c_{3} v_{3}+c_{4} v_{4}$ then conditions (2.5) are a system of equations in the constants $c_{1}, c_{2}, c_{3}, c_{4}$ whose determinant is the Gram determinant of the linearly independent functions $v_{1}, v_{2}, v_{3}, v_{4}$ and is therefore nonzero. Let $y(x)$ denote the solution of $l(y)=f$ satisfying the initial conditions $y(0)=\alpha_{0}, y^{\prime}(0)=\alpha_{1}, y^{\prime \prime}(0)=\alpha_{2}, y^{\prime \prime \prime}(0)=\alpha_{3}$. We suppose that $y(x)$ is the desired element. Applying Green' formula to $y(x)$ and $v_{j}$, we get

$$
\left(f, v_{j}\right)_{L^{2}}=\left(l(y), v_{j}\right)_{L^{2}}=\left[y, v_{j}\right]_{\infty}-\left[y, v_{j}\right]_{0}, j=1,2,3,4 .
$$

But $l\left(v_{j}\right)=0(j=1,2,3,4)$. Since $y(0)=\alpha_{0}, y^{\prime}(0)=\alpha_{1}, y^{\prime \prime}(0)=$ $\alpha_{2}, y^{\prime \prime \prime}(0)=\alpha_{3}$, we have

$$
\left[y, v_{j}\right]_{0}=\left\{\begin{array}{cc}
\alpha_{3}, & \text { for } j=1 \\
-\alpha_{2}, & \text { for } j=2 \\
\alpha_{1}, & \text { for } j=3 \\
-\alpha_{0}, & \text { for } j=4
\end{array}\right\} .
$$

Hence

$$
\begin{aligned}
& \left(f, v_{1}\right)_{L^{2}}=\left[y, v_{1}\right]_{\infty}-\alpha_{3},\left(f, v_{2}\right)_{L^{2}}=\left[y, v_{2}\right]_{\infty}+\alpha_{2} \\
& \left(f, v_{3}\right)_{L^{2}}=\left[y, v_{3}\right]_{\infty}-\alpha_{1},\left(f, v_{4}\right)_{L^{2}}=\left[y, v_{4}\right]_{\infty}+\alpha_{0}
\end{aligned}
$$

According to (2.5), we have

$$
\left[y, v_{1}\right]_{\infty}=\beta_{0},\left[y, v_{2}\right]_{\infty}=\beta_{1},\left[y, v_{3}\right]_{\infty}=\beta_{2},\left[y, v_{4}\right]_{\infty}=\beta_{3} .
$$

We recall that a triple $\left(\mathbb{H}, \Gamma_{1}, \Gamma_{2}\right)$ is called a space of boundary values of a closed symmetric operator $A$ on a Hilbert space $H$ if $\Gamma_{1}, \Gamma_{2}$ are linear maps from $D\left(A^{*}\right)$ to $\mathbb{H}$ with equal deficiency numbers such that:

i) Green's formula is valid

$$
\left(A^{*} f, g\right)_{H}-\left(f, A^{*} g\right)_{H}=\left(\Gamma_{1} f, \Gamma_{2} g\right)_{\mathbb{H}}-\left(\Gamma_{2} f, \Gamma_{1} g\right)_{\mathbb{H}}, f, g \in D\left(A^{*}\right) .
$$

ii) For any $F_{1}, F_{2} \in H$, there is a vector $f \in D\left(A^{*}\right)$ such that $\Gamma_{1} f=$ $F_{1}, \Gamma_{2} f=F_{2}([14],[15])$. 
Theorem 1. The triple $\left(\mathbb{C}^{4}, \Gamma_{1}, \Gamma_{2}\right)$ defined by (2.2) is a boundary spaces of the operator $L_{0}$.

Proof. First condition of the definition of a space of boundary value follows from Lemma 1 and second condition follows from Lemma 2.

Corollary 1. For any contraction $K$ in $\mathbb{C}^{4}$ the restriction of the operator $L$ to the set of functions $y \in D$ satisfying either

$$
(K-I) \Gamma_{1} y+i(K+I) \Gamma_{2} y=0
$$

or

$$
(K-I) \Gamma_{1} y-i(K+I) \Gamma_{2} y=0
$$

is respectively the maximal dissipative and accretive extension of the operator $L_{0}$. Conversely, every maximal dissipative (accretive) extension of the operator $L_{0}$ is the restriction of $L$ to the set of functions $y \in D$ satisfying (2.6) ( (2.7) ), and the extension uniquely determines the contraction K. Conditions (2.6) ((2.7)), in which $K$ is an isometry describe the maximal symmetric extensions of $L_{0}$ in $L_{2}(0, \infty)$. If $K$ is unitary, these conditions define selfadjoint extensions.

In particular, the boundary conditions

$$
\begin{gathered}
y^{\prime \prime \prime}(0)+h_{1} y(0)=0, \\
y^{\prime}(0)+h_{2} y^{\prime \prime}(0)=0, \\
{\left[y, v_{2}\right]_{\infty}-h_{3}\left[y, v_{4}\right]_{\infty}=0,} \\
{\left[y, v_{1}\right]_{\infty}-h_{4}\left[y, v_{3}\right]_{\infty}=0}
\end{gathered}
$$

with $\operatorname{Im} h_{1} \geq 0$ or $h_{1}=\infty, \operatorname{Im} h_{2} \geq 0$ or $h_{2}=\infty, \operatorname{Im} h_{3} \geq 0$ or $h_{3}=\infty$ and $\operatorname{Im} h_{4} \geq 0$ or $h_{4}=\infty\left(\operatorname{Im} h_{1}=0\right.$ or $h_{1}=\infty, \operatorname{Im} h_{2}=0$ or $h_{2}=\infty, \operatorname{Im} h_{3}=0$ or $h_{3}=\infty$ and $\operatorname{Im} h_{4}=0$ or $h_{4}=\infty$ ) describe the maximal dissipative (selfadjoint) extensions of $L_{0}$ with separated boundary conditions.

Now, we study the maximal dissipative operator $L_{K}$, where $K$ is the strict contraction in $\mathbb{C}^{4}$ generated by the expression $l(y)$ and boundary condition (2.6).

Let us define the "incoming" and "outgoing" subspaces $D_{-}=L^{2}(-\infty, 0)$ and $D_{+}=L^{2}(0, \infty)$. The orthogonal sum $\mathrm{H}=D_{-} \oplus H \oplus D_{+}$is called main Hilbert space of the dilation.

In the space $\mathcal{H}$, we consider the operator $\mathcal{L}_{G}$ on the set $D\left(\mathcal{L}_{G}\right)$, its elements consisting of vectors $w=\left\langle\varphi_{-}, y, \varphi_{+}\right\rangle$, generated by the expression

$$
\mathcal{L}_{G}\left\langle\varphi_{-}, \widehat{y}, \varphi_{+}\right\rangle=\left\langle i \frac{d \varphi_{-}}{d \xi}, l(y), i \frac{d \varphi_{+}}{d \xi}\right\rangle
$$


satisfying the conditions: $\varphi_{-} \in W_{2}^{1}(-\infty, 0), \varphi_{+} \in W_{2}^{1}(0, \infty), y \in H, y^{\prime \prime \prime}(0)+$ $h_{1} y(0)=0, y^{\prime}(0)+h_{2} y^{\prime \prime}(0)=0, \quad\left[y, v_{2}\right]_{\infty}-h_{3}\left[y, v_{4}\right]_{\infty}=0, \quad\left[y, v_{1}\right]_{\infty}-$ $G\left[y, v_{3}\right]_{\infty}=C \varphi_{-}(0),\left[y, v_{1}\right]_{\infty}-\bar{G}\left[y, v_{3}\right]_{\infty}=C \varphi_{+}(0)$, where $W_{2}^{1}$ are Sobolev spaces and $C^{2}:=2 \operatorname{Im} G, C>0$.

Theorem 2. The operator $\mathcal{L}_{G}$ is selfadjoint in $\mathcal{H}$ and it is a selfadjoint dilation of the operator $\widetilde{L}_{G}\left(=L_{K}\right)$.

Proof. Let $f, g \in D\left(\mathcal{L}_{G}\right), f=\left\langle\varphi_{-}, y, \varphi_{+}\right\rangle$and $g=\left\langle\psi_{-}, z, \psi_{+}\right\rangle$. Then we have

$$
\begin{aligned}
& \left(\mathcal{L}_{G} f, g\right)_{\mathcal{H}}-\left(f, \mathcal{L}_{G} g\right)_{\mathcal{H}}=\left(\mathcal{L}_{G}\left\langle\varphi_{-}, y, \varphi_{+}\right\rangle,\left\langle\psi_{-}, z, \psi_{+}\right\rangle\right) \\
& -\left(\left\langle\varphi_{-}, y, \varphi_{+}\right\rangle, \mathcal{L}_{G}\left\langle\psi_{-}, z, \psi_{+}\right\rangle\right) \\
& =\int_{-\infty}^{0} i \varphi_{-}^{\prime} \bar{\psi}_{-} d \xi+(l(y), z)_{H}+\int_{0}^{\infty} i \varphi_{+}^{\prime} \bar{\psi}_{+} d \xi \\
& -\int_{-\infty}^{0} i \psi_{-}^{\prime} \bar{\varphi}_{-} d \xi-(y, l(z))_{H}-\int_{0}^{\infty} i \psi_{+}^{\prime} \bar{\varphi}_{+} d \xi \\
& =\int_{-\infty}^{0} i \varphi_{-}^{\prime} \bar{\psi}_{-} d \xi+\left[y, \bar{z}_{\infty}+\int_{0}^{\infty} i \varphi_{+}^{\prime} \bar{\psi}_{+} d \xi\right. \\
& -\int_{-\infty}^{0} i \psi_{-}^{\prime} \bar{\varphi}_{-} d \xi-[y, \bar{z}]_{0}-\int_{0}^{\infty} i \psi_{+}^{\prime} \bar{\varphi}_{+} d \xi \\
& =i \psi_{-}(0) \bar{\varphi}_{-}(0)-i \varphi_{+}(0) \bar{\psi}_{+}(0)+[y, \bar{z}]_{\infty}-[y, \bar{z}]_{0} .
\end{aligned}
$$

By direct computation, we obtain

$$
i \psi_{-}(0) \bar{\varphi}_{-}(0)-i \varphi_{+}(0) \bar{\psi}_{+}(0)+[y, \bar{z}]_{\infty}-[y, \bar{z}]_{0}=0 .
$$

Thus, $\mathcal{L}_{G}$ is a symmetric operator. If we show that $\mathcal{L}_{G} \subseteq \mathcal{L}_{G}^{*}$, we prove that $\mathcal{L}_{G}$ is selfadjoint. Take $g=\left\langle\psi_{-}, z, \psi_{+}\right\rangle \in D\left(\mathcal{L}_{G}^{*}\right)$. Let $\mathcal{L}_{G}^{*} g=g^{*}=$ $\left\langle\psi_{-}^{*}, z^{*}, \psi_{+}^{*}\right\rangle \in \mathcal{H}$, so that

$$
\left(\mathcal{L}_{G} f, g\right)_{\mathcal{H}}=\left(f, \mathcal{L}_{G}^{*} g\right)_{\mathcal{H}}=\left(f, g^{*}\right)_{\mathcal{H}} .
$$

It is easy to show that $\psi_{-} \in W_{2}^{1}(-\infty, 0), \psi_{+} \in W_{2}^{1}(0, \infty), g \in D\left(\mathcal{L}_{G}\right)$ and $g^{*}=\mathcal{L}_{G} g$, by choosing elements with suitable components as the $f \in D\left(\mathcal{L}_{G}\right)$ in (2.13). Then we have

$$
\left(\mathcal{L}_{G} f, g\right)_{\mathcal{H}}=\left(f, \mathcal{L}_{G} g\right)_{\mathcal{H}}
$$


for all $f \in D\left(\mathcal{L}_{G}^{*}\right)$. Furthermore, $g \in D\left(\mathcal{L}_{G}^{*}\right)$ satisfies the conditions

$$
\begin{aligned}
{\left[y, v_{1}\right]_{\infty}-G\left[y, v_{3}\right]_{\infty} } & =C \varphi_{-}(0), \\
{\left[y, v_{1}\right]_{\infty}-\bar{G}\left[y, v_{3}\right]_{\infty} } & =C \varphi_{+}(0) .
\end{aligned}
$$

Hence, $D\left(\mathcal{L}_{G}^{*}\right) \subseteq D\left(\mathcal{L}_{G}\right)$, i.e., $\mathcal{L}_{G}=\mathcal{L}_{G}^{*}$.

The selfadjoint operator $\mathcal{L}_{G}$ generates on $\mathcal{H}$ a unitary group $U_{t}=\exp \left(i \mathcal{L}_{G} t\right)\left(t \in \mathbb{R}_{+}=(0, \infty)\right)$. Let denote by $P: \mathcal{H} \rightarrow H$ and $P_{1}: H \rightarrow \mathcal{H}$ the mapping acting according to the formulae $P:\left\langle\varphi_{-}, y, \varphi_{+}\right\rangle \rightarrow \widehat{y}$ and $P_{1}: y \rightarrow\langle 0, y, 0\rangle$. Let $Z_{t}:=P U_{t} P_{1}, t \geq 0$, by using $U_{t}$. The family $\left\{Z_{t}\right\}(t \geq 0)$ of operators is a strongly continuous semigroup of completely nonunitary contraction on $H$. Let us denote by $B_{G}$ the generator of this semigroup: $B_{G} y=\lim _{t \rightarrow+0}(i t)^{-1}\left(Z_{t} y-y\right)$. The domain of $B_{G}$ consists of all the vectors for which the limit exists. The operator $B_{G}$ is dissipative. The operator $\mathcal{L}_{G}$ is called the selfadjoint dilation of $B_{G}$ (see $\left.[5,16,19]\right)$. We show that $B_{G}=\widetilde{L}_{G}$, hence $\mathcal{L}_{G}$ is selfadjoint dilation of $B_{G}$. To show this, it is sufficient to verify the equality

$$
P\left(\mathcal{L}_{G}-\lambda I\right)^{-1} P_{1} y=\left(\widetilde{L}_{G}-\lambda I\right)^{-1} y, y \in H, \operatorname{Im} h<0 .
$$

For this purpose, we set $\left(\mathcal{L}_{G}-\lambda I\right)^{-1} P_{1} y=g=\left\langle\psi_{-}, z, \psi_{+}\right\rangle$implies that $\left(\mathcal{L}_{G}-\lambda I\right) g=P_{1} y$, and hence $l(z)-\lambda z=y, \psi_{-}(\xi)=\psi_{-}(0) e^{-i \lambda \xi}$ and $\psi_{+}(\xi)=\psi_{+}(0) e^{-i \lambda \xi}$. Since $g \in D\left(\mathcal{L}_{G}\right)$, then $\psi_{-} \in W_{2}^{1}(-\infty, 0)$, it follows that $\psi_{-}(0)=0$, and consequently $z$ satisfies the boundary condition $\left[y, v_{1}\right]_{\infty}-$ $G\left[y, v_{3}\right]_{\infty}=0$. Therefore $z \in D\left(\widetilde{L}_{G}\right)$, and since point $\lambda$ with $\operatorname{Im} \lambda<0$ cannot be an eigenvalue of dissipative operator, then $z=\left(\widetilde{L}_{G}-\lambda I\right)^{-1} y$. Thus

$$
\left(\mathcal{L}_{G}-\lambda I\right)^{-1} P_{1} y=\left\langle 0,\left(\widetilde{L}_{G}-\lambda I\right)^{-1} y, C^{-1}\left(\left[y, v_{1}\right]_{\infty}-\bar{G}\left[y, v_{3}\right]_{\infty}\right) e^{-i \lambda \xi}\right\rangle
$$

for $y$ and $\operatorname{Im} \lambda<0$. On applying the mapping $P$, we obtain (2.14), and

$$
\begin{aligned}
\left(\widetilde{L}_{G}-\lambda I\right)^{-1} & =P\left(\mathcal{L}_{G}-\lambda I\right)^{-1} P_{1}=-i P \int_{0}^{\infty} U_{t} e^{-i \lambda t} d t P_{1} \\
& =-i \int_{0}^{\infty} Z_{t} e^{-i \lambda t} d t=\left(B_{G}-\lambda I\right)^{-1}, \operatorname{Im} \lambda<0
\end{aligned}
$$

so this clearly shows that $\widetilde{L}_{G}=B_{G}$. 


\section{Functional Model of Dissipative Fourth Order Opera- tor}

The unitary group $\left\{U_{t}\right\}$ has an important property which makes it possible to apply it to the Lax-Phillips [18]. It has orthogonal incoming and outgoing subspaces $D_{-}=\left\langle L^{2}(-\infty, 0), 0,0\right\rangle$ and $D_{+}=\left\langle 0,0, L^{2}(0, \infty)\right\rangle$ having the following properties:

(1) $U_{t} D_{-} \subset D_{-}, t \leq 0$ and $U_{t} D_{+} \subset D_{+}, t \geq 0$;

(2) $\cap U_{t} D_{-}=\cap \bar{U}_{t} D_{+}=\{0\}$;

(3) $\frac{t \leq 0}{\bigcup_{t \geq 0} U_{t} D_{-}}=\frac{t \geq 0}{\bigcup_{t \leq 0} U_{t} D_{+}}=\mathcal{H}$

(4) $D_{-} \perp D_{+}$.

Property (4) is clear. To be able to prove property (1) for $D_{+}$(the proof for $D_{-}$is similar), we set $\mathcal{R}_{\lambda}=\left(\mathcal{L}_{G}-\lambda I\right)^{-1}$. For all $\lambda$, with $\operatorname{Im} \lambda<0$ and for any $f=\left\langle 0,0, \varphi_{+}\right\rangle \in D_{+}$, we have

$$
\mathcal{R}_{\lambda} f=\left\langle 0,0,-i e^{-i \lambda \xi} \int_{0}^{\xi} e^{i \lambda s} \varphi_{+}(s) d s\right\rangle .
$$

As $\mathcal{R}_{\lambda} f \in D_{+}$. Therefore, if $g \perp D_{+}$, then

$$
0=\left(\mathcal{R}_{\lambda} f, g\right)_{\mathcal{H}}=-i \int_{0}^{\infty} e^{-i \lambda t}\left(U_{t} f, g\right)_{\mathcal{H}} d t, \quad \operatorname{Im} \lambda<0
$$

which implies that $\left(U_{t} f, g\right)_{\mathcal{H}}=0$ for all $t \geq 0$. Hence, for $t \geq 0, U_{t} D_{+} \subset D_{+}$, and property (1) has been proved.

In order to prove property (2), we define the mappings $P^{+}: \mathcal{H} \rightarrow L^{2}(0, \infty)$ and $P_{1}^{+}: L^{2}(0, \infty) \rightarrow D_{+}$as follows $P^{+}:\left\langle\varphi_{-}, \widehat{y}, \varphi_{+}\right\rangle \rightarrow \varphi_{+}$and $P_{1}^{+}: \varphi \rightarrow$ $\langle 0,0, \varphi\rangle$, respectively. We take into consider that the semigroup of isometries $U_{t}^{+}:=P^{+} U_{t} P_{1}^{+} \quad(t \geq 0)$ is a one-sided shift in $L^{2}(0, \infty)$. Indeed, the generator of the semigroup of the one-sided shift $V_{t}$ in $L^{2}(0, \infty)$ is the differential operator $i\left(\frac{d}{d \xi}\right)$ with the boundary condition $\varphi(0)=0$. On the other hand, the generator $S$ of the semigroup of isometries $U_{t}^{+}(t \geq 0)$ is the operator $S \varphi=P^{+} \mathcal{L}_{G} P_{1}^{+} \varphi=P^{+} \mathcal{L}_{G}\langle 0,0, \varphi\rangle=P^{+}\left\langle 0,0, i\left(\frac{d}{d \xi}\right) \varphi\right\rangle=i\left(\frac{d}{d \xi}\right) \varphi$, where $\varphi \in W_{2}^{1}(0, \infty)$ and $\varphi(0)=0$. Since a semigroup is uniquely determined by its generator, it follows that $U_{t}^{+}=V_{t}$, and, hence,

$$
\bigcap_{t \geq 0} U_{t} D_{+}=\left\langle 0,0, \cap_{t \leq 0} V_{t} L^{2}(0, \infty)\right\rangle=\{0\}
$$

so the proof is completed. 
Definition 1. The linear operator $A$ with domain $D(A)$ acting in the Hilbert space $H$ is called completely nonselfadjoint (or simple) if there is no invariant subspace $M \subseteq D(A)(M \neq\{0\})$ of the operator $A$ on which the restriction A to $M$ is selfadjoint.

To prove property (3) of the incoming and outgoing subspaces, let us prove following lemma.

Lemma 3. The operator $\widetilde{L}_{G}$ is completely noselfadjoint (simple).

Proof. Let $H^{\prime} \subset H$ be a nontrivial subspace in which $\widetilde{L}_{G}$ induces a selfadjoint operator $\widetilde{L}_{G}^{\prime}$ with domain $D\left(\widetilde{L}_{G}^{\prime}\right)=H^{\prime} \cap D\left(\widetilde{L}_{G}\right)$. If $f \in D\left(\widetilde{L}_{G}^{\prime}\right)$, then $f \in D\left(\widetilde{L}_{G}^{*}\right)$ and

$$
\begin{aligned}
0 & =\frac{d}{d t}\left\|e^{i \widetilde{L}_{G} t} f\right\|_{H}^{2}=\frac{d}{d t}\left(e^{i \widetilde{L}_{G} t} f, e^{i \widetilde{L}_{G} t} f\right)_{H} \\
& =-C^{2}\left(\left[e^{i \widetilde{L}_{G} t} f, v_{3}\right]\right) .
\end{aligned}
$$

Consequently, we have $\left[e^{i \widetilde{L}_{G}^{\prime} t} f, v_{3}\right]=0$. Using this result with boundary condition $\left[y, v_{1}\right]_{\infty}-G\left[y, v_{3}\right]_{\infty}=0$, we have $\left[y, v_{1}\right]_{\infty}=0$, i.e., $y(\lambda)=0$. Since all solutions of $l(y)=\lambda y$ belong to $L^{2}(0, \infty)$, from this it can be concluded that the resolvent $R_{\lambda}\left(\widetilde{L}_{G}\right)$ is a compact operator, and the spectrum of $\widetilde{L}_{G}$ is purely discrete. Consequently, by the theorem on expansion in the eigenvectors of the selfadjoint operator $\widetilde{L}_{G}^{\prime}$, we obtain $H^{\prime}=\{0\}$. Hence the operator $\widetilde{L}_{G}$ is simple. The proof is completed.

Let us define $H_{-}=\overline{\bigcup_{t \geq 0} U_{t} D_{-}}, H_{+}=\overline{\bigcup_{t \leq 0} U_{t} D_{+}}$.

Lemma 4. The equality $H_{-}+H_{+}=\mathcal{H}$ holds.

Proof. Considering property (1) of the subspace $D_{+}$, it is easy to show that the subspace $\mathcal{H}^{\prime}=\mathcal{H} \odot\left(H_{-}+H_{+}\right)$is invariant relative to the group $\left\{U_{t}\right\}$ and has the form $\mathcal{H}^{\prime}=\left\langle 0, H^{\prime}, 0\right\rangle$, where $H^{\prime}$ is a subspace in $H$. Therefore, if the subspace $\mathcal{H}^{\prime}$ (and hence also $H^{\prime}$ ) were nontrivial, then the unitary group $\left\{U_{t}\right\}$ restricted to this subspace would be a unitary part of the group $\left\{U_{t}\right\}$, and hence, the restriction $\widetilde{L}_{G}^{\prime}$ of $\widetilde{L}_{G}$ to $H^{\prime}$ would be a selfadjoint operator in $H^{\prime}$. Since the operator $\widetilde{L}_{G}$ is simple, it follows that $H^{\prime}=\{0\}$. The lemma is proved. 
Assume that $\varphi(x, \lambda)$ and $\psi(x, \lambda)$ are solutions of $l(y)=\lambda y$ satisfying the conditions

$$
\begin{aligned}
\varphi^{\prime}(0, \lambda) & =1, \varphi(0, \lambda)=0, \psi^{\prime}(0, \lambda)=0, \psi(0, \lambda)=1, \\
{\left[\varphi, v_{4}\right]_{\infty} } & =-\frac{1}{\sqrt{1+h_{3}^{2}}},\left[\varphi, v_{2}\right]_{\infty}=\frac{h_{3}}{\sqrt{1+h_{3}^{2}}}, \\
{\left[\psi, v_{4}\right]_{\infty} } & =\frac{h_{3}}{\sqrt{1+h_{3}^{2}}},\left[\psi, v_{2}\right]_{\infty}=\frac{1}{\sqrt{1+h_{3}^{2}}} .
\end{aligned}
$$

Let us adopt the following notations:

$$
\begin{gathered}
k(\lambda):=-\frac{\left[\varphi, v_{1}\right]_{\infty}}{\left[\psi, v_{3}\right]_{\infty}}, M(\lambda)=-\frac{\left[\psi, v_{3}\right]_{\infty}}{\left[\varphi, v_{3}\right]_{\infty}}, \\
S_{G}(\lambda)=\frac{M(\lambda) k(\lambda)-G}{M(\lambda) k(\lambda)-\bar{G}} .
\end{gathered}
$$

$M(\lambda)$ is a meromorphic function on the complex plane $\mathbb{C}$ with a countable number of poles on the real axis. Further, it is possible to show that the function $M(\lambda)$ possesses the following properties: $\operatorname{Im} M(\lambda) \leq 0$ for all $\operatorname{Im} \lambda \neq$ 0 , and $M^{*}(\lambda)=M(\bar{\lambda})$ for all $\lambda \in \mathbb{C}$, except the real poles $M(\lambda)$.

We set

$$
\begin{gathered}
U_{\lambda}^{-}(x, \xi, \zeta)= \\
\left\langle e^{-i \lambda \xi}, \alpha M(\lambda)\left[(M(\lambda) k(\lambda)-G)\left[\psi, v_{3}\right]_{\infty}\right]^{-1} \overline{\varphi(x, \lambda)}, \overline{S_{G}}(\lambda) e^{-i \lambda \zeta}\right\rangle .
\end{gathered}
$$

We note that the vectors $U_{\lambda}^{-}(x, \xi, \zeta)$ for real $\lambda$ do not belong to the space $\mathcal{H}$. However, $U_{\lambda}^{-}(x, \xi, \zeta)$ satisfies the equation $\mathcal{L} U=\lambda U$ and the corresponding boundary conditions for the operator $\mathcal{L}_{h}$.

By means of vector $U_{\lambda}^{-}(x, \xi, \zeta)$, we define the transformation $F_{-}: f \rightarrow$ $\tilde{f_{-}}(\lambda)$ by

$$
\left(F_{-} f\right)(\lambda):=\tilde{f}_{-}(\lambda):=\frac{1}{\sqrt{2 \pi}}\left(f, U_{\bar{\lambda}}\right)_{\mathcal{H}}
$$

on the vectors $f=\left\langle\varphi_{-}, \widehat{y}, \varphi_{+}\right\rangle$in which $\varphi_{-}(\xi), \varphi_{+}(\zeta), y(x)$ are smooth, compactly supported functions

Lemma 5. The transformation $F_{-}$isometrically maps $H_{-}$onto $L^{2}(\mathbb{R})$. For all vectors $f, g \in H_{-}$the Parseval equality and the inversion formulae hold:

$$
(f, g)_{\mathcal{H}}=\left(\tilde{f_{-}}, \tilde{g_{-}}\right)_{L^{2}}=\int_{-\infty}^{\infty} \tilde{f_{-}}(\lambda) \overline{\tilde{g_{-}}(\lambda)} d \lambda, f=\frac{1}{\sqrt{2 \pi}} \int_{-\infty}^{\infty} \tilde{f_{-}}(\lambda) U_{\bar{\lambda}} d \lambda,
$$

where $\tilde{f}_{-}(\lambda)=\left(F_{-} f\right)(\lambda)$ and $\tilde{g}_{-}(\lambda)=\left(F_{-} g\right)(\lambda)$. 
Proof. For $f, g \in D_{-}, f=\left\langle\varphi_{-}, 0,0\right\rangle, g=\left\langle\psi_{-}, 0,0\right\rangle$, with Paley-Wiener theorem, we have

$$
\tilde{f_{-}}(\lambda)=\frac{1}{\sqrt{2 \pi}}\left(f, U_{\bar{\lambda}}\right)_{\mathcal{H}}=\frac{1}{\sqrt{2 \pi}} \int_{-\infty}^{0} \varphi_{-}(\xi) e^{-i \lambda \xi} d \xi \in H_{-}^{2}
$$

and by using usual Parseval equality for Fourier integrals,

$$
(f, g)_{\mathcal{H}}=\int_{-\infty}^{\infty} \varphi_{-}(\xi) \overline{\psi_{-}(\xi)} d \xi=\int_{-\infty}^{\infty} \tilde{f_{-}}(\lambda) \overline{\tilde{g_{-}}(\lambda)} d \lambda=\left(F_{-} f, F_{-} g\right)_{L^{2}}
$$

Here, $H_{ \pm}^{2}$ denote the Hardy classes in $L^{2}(\mathbb{R})$ consisting of the functions analytically extendible to the upper and lower half-planes, respectively.

We now extend to the Parseval equality to the whole of $H_{-}$. We consider in $H_{-}$the dense set of $H_{-}^{\prime}$ of the vectors obtained as follows from the smooth, compactly supported functions in $D_{-}: f \in H_{-}^{\prime}$ if $f=U_{T} f_{0}$, $f_{0}=\left\langle\varphi_{-}, 0,0\right\rangle, \varphi_{-} \in C_{0}^{\infty}(-\infty, 0)$, where $T=T_{f}$ is a nonnegative number depending on $f$. If $f, g \in H_{-}^{\prime}$, then for $T>T_{f}$ and $T>T_{g}$ we have $U_{-T} f, U_{-T} g \in D_{-}$, moreover, the first components of these vectors belong to $C_{0}^{\infty}(-\infty, 0)$. Therefore, since the operators $U_{t}(t \in \mathbb{R})$ are unitary, by the equality

$$
F_{-} U_{t} f=\left(U_{t} f, U_{\lambda}^{-}\right)_{\mathcal{H}}=e^{i \lambda t}\left(f, U_{\lambda}^{-}\right)_{\mathcal{H}}=e^{i \lambda t} F_{-} f
$$

we have

$$
(f, g)_{\mathcal{H}}=\left(U_{-T} f, U_{-T} g\right)_{\mathcal{H}}=\left(F_{-} U_{-T} f, F_{-} U_{-T} g\right)_{L^{2}}
$$

and

$$
\left(e^{i \lambda T} F_{-} f, e^{i \lambda T} F_{-} g\right)_{L^{2}}=(\tilde{f}, \tilde{g})_{L^{2}} .
$$

By taking the closure (3.2), we obtain the Parseval equality for the space $H_{-}$. The inversion formula is obtained from the Parseval equality if all integrals in it are considered as limits in the of integrals over finite intervals. Finally $F_{-} H_{-}=\overline{\bigcup_{t \geq 0} F_{-} U_{t} D_{-}}=\overline{\bigcup_{t \geq 0} e^{i \lambda t} H_{-}^{2}}=L^{2}(\mathbb{R})$, that is $F_{-}$maps $H_{-}$onto the whole of $L^{2}(\mathbb{R})$. The lemma is proved.

We set

$$
\begin{gathered}
U_{\lambda}^{+}(x, \xi, \zeta)= \\
\left\langle S_{G}(\lambda) e^{-i \lambda \xi}, \alpha M(\lambda)\left[(M(\lambda) k(\lambda)-\bar{G})\left[\psi, v_{3}\right]_{\infty}\right]^{-1} \varphi(x, \lambda), e^{-i \lambda \zeta}\right\rangle .
\end{gathered}
$$


We note that the vectors $U_{\lambda}^{+}(x, \xi, \zeta)$ for real $\lambda$ do not belong to the space $\mathcal{H}$. However, $U_{\lambda}^{+}(x, \xi, \zeta)$ satisfies the equation $\mathcal{L} U=\lambda U$ and the corresponding boundary conditions for the operator $\mathcal{L}_{h}$. With the help of vector $U_{\lambda}^{+}(x, \xi, \zeta)$, we define the transformation $F_{+}: f \rightarrow \tilde{f_{+}}(\lambda)$ by $\left(F_{+} f\right)(\lambda):=\tilde{f_{+}}(\lambda):=$ $\frac{1}{\sqrt{2 \pi}}\left(f, U_{\lambda}^{+}\right)_{\mathcal{H}}$ on the vectors $f=\left\langle\varphi_{-}, \widehat{y}, \varphi_{+}\right\rangle$in which $\varphi_{-}(\xi), \varphi_{+}(\zeta)$ and $y(x)$ are smooth, compactly supported functions.

Lemma 6. The transformation $F_{+}$isometrically maps $H_{+}$onto $L^{2}(\mathbb{R})$. For all vectors $f, g \in H_{+}$the Parseval equality and the inversion formula hold:

$$
(f, g)_{\mathcal{H}}=\left(\tilde{f_{+}}, \tilde{g_{+}}\right)_{L^{2}}=\int_{-\infty}^{\infty} \tilde{f_{+}}(\lambda) \overline{\tilde{g_{+}}(\lambda)} d \lambda, \quad f=\frac{1}{\sqrt{2 \pi}} \int_{-\infty}^{\infty} \tilde{f_{+}}(\lambda) U_{\lambda}^{+} d \lambda
$$

where $\tilde{f_{+}}(\lambda)=\left(F_{+} f\right)(\lambda)$ and $\tilde{g_{+}}(\lambda)=\left(F_{+} g\right)(\lambda)$.

Proof. The proof is analogous to the Lemma 5.

It is obvious that the matrix-valued function $S_{G}(\lambda)$ is meromorphic in $\mathbb{C}$ and all poles are in the lower half-plane. From (3.1), $\left|S_{G}(\lambda)\right| \leq 1$ for $\operatorname{Im} \lambda>0$; and $S_{G}(\lambda)$ is the unitary matrix for all $\lambda \in \mathbb{R}$. Therefore, it explicitly follows from the formulae for the vectors $U_{\lambda}^{-}$and $U_{\lambda}^{+}$that

$$
U_{\lambda}^{+}=S_{G}(\lambda) U_{\lambda}^{-} .
$$

It follows from Lemmas 5 and 6 that $H_{-}=H_{+}$. Together with Lemma 4 , this shows that $H_{-}=H_{+}=\mathcal{H}$, therefore property (3) above has been proved for the incoming and outgoing subspaces.

Thus, the transformation $F_{-}$isometrically maps $H_{-}$onto $L^{2}(\mathbb{R})$ with the subspace $D_{-}$mapped onto $H_{-}^{2}$ and the operators $U_{t}$ are transformed into the operators of multiplication by $e^{i \lambda t}$. This means that $F_{-}$is the incoming spectral representation for the group $\left\{U_{t}\right\}$. Similarly, $F_{+}$is the outgoing spectral representation for the group $\left\{U_{t}\right\}$. It follows from (3.3) that the passage from the $F_{-}$representation of an element $f \in \mathcal{H}$ to its $F_{+}$representation is accomplished as $\tilde{f_{+}}(\lambda)=S_{G}^{-1}(\lambda) \tilde{f_{-}}(\lambda)$. Consequently, according to [18], we have proved the following.

Theorem 3. The function $\overline{S_{G}}(\lambda)$ is the scattering matrix of the group $\left\{U_{t}\right\}$ (of the selfadjoint operator $\mathcal{L}_{G}$ ).

Let $S(\lambda)$ be an arbitrary nonconstant inner function (see [19]) on the upper half-plane (the analytic function $S(\lambda)$ on the upper half-plane $\mathbb{C}_{+}$is called inner function on $\mathbb{C}_{+}$if $\left|S_{h}(\lambda)\right| \leq 1$ for all $\lambda \in \mathbb{C}_{+}$and $\left|S_{h}(\lambda)\right|=1$ for 
almost all $\lambda \in \mathbb{R}$ ). Define $K=H_{+}^{2} \ominus S H_{+}^{2}$. Then $K \neq\{0\}$ is a subspace of the Hilbert space $H_{+}^{2}$. We consider the semigroup of operators $Z_{t}(t \geq 0)$ acting in $K$ according to the formula $Z_{t} \varphi=P\left[e^{i \lambda t} \varphi\right], \varphi=\varphi(\lambda) \in K$, where $P$ is the orthogonal projection from $H_{+}^{2}$ onto $K$. The generator of the semigroup $\left\{Z_{t}\right\}$ is denoted by

$$
T \varphi=\lim _{t \rightarrow+0}(i t)^{-1}\left(Z_{t} \varphi-\varphi\right),
$$

which $T$ is a maximal dissipative operator acting in $K$ and with the domain $D(T)$ consisting of all functions $\varphi \in K$, such that the limit exists. The operator $T$ is called a model dissipative operator. Recall that this model dissipative operator, which is associated with the names of Lax-Phillips [18], is a special case of a more general model dissipative operator constructed by Nagy and Foiaş [19]. The basic assertion is that $S(\lambda)$ is the characteristic function of the operator $T$.

Let $K=\langle 0, H, 0\rangle$, so that $\mathcal{H}=D_{-} \oplus K \oplus D_{+}$. It follows from the explicit form of the unitary transformation $F_{-}$under the mapping $F_{-}$

$$
\begin{aligned}
& \mathcal{H} \rightarrow L^{2}(\mathbb{R}), f \rightarrow \tilde{f_{-}}(\lambda)=\left(F_{-} f\right)(\lambda), D_{-} \rightarrow H_{-}^{2}, D_{+} \rightarrow S_{G} H_{+}^{2}, \\
& K \rightarrow H_{+}^{2} \ominus S_{G} H_{+}^{2}, U_{t} \rightarrow\left(F_{-} U_{t} F_{-}^{-1} \tilde{f_{-}}\right)(\lambda)=e^{i \lambda t} \tilde{f_{-}}(\lambda) .
\end{aligned}
$$

The formulas (3.4) show that operator $\widetilde{L}_{G}$ is a unitarily equivalent to the model dissipative operator with the characteristic function $S_{G}(\lambda)$. We have thus proved following theorem.

Theorem 4. The characteristic function of the maximal dissipative operator $\widetilde{L}_{G}$ coincides with the function $S_{G}(\lambda)$ defined (3.1).

\section{The spectral properties of dissipative fourth order op- erators}

Using characteristic function, we investigate the spectral properties of the maximal dissipative operator $\widetilde{L}_{G}\left(L_{K}\right)$. We know that the characteristic function of a maximal dissipative operator carries information about the spectral properties of this operator. In order to prove completeness of the system of eigenvectors and associated vectors of the operator $\widetilde{L}_{G}\left(L_{K}\right)$ in the space $L_{2}(0, \infty)$ (see $\left.[5,16,19]\right)$, we must show that there exists no singular factor $s(\lambda)$ of the characteristic funcion $S_{G}(\lambda)$ in the factorization $\operatorname{det} S_{G}(\lambda)=$ $s(\lambda) B(\lambda)(B(\lambda)$ is a Blaschke product) (see $[3,22,25])$. 
The characteristic function $S_{G}(\lambda)$ of the maximal dissipative operator $\widetilde{L}_{G}$ has the form

$$
S_{G}(\lambda):=\frac{M(\lambda) k(\lambda)-G}{M(\lambda) k(\lambda)-\bar{G}}
$$

where $\operatorname{Im} G>0$.

Theorem 5. For all the values of $G$ with $\operatorname{Im} G>0$, except possibly for a single value $G=G_{0}$, the characteristic function $S_{G}(\lambda)$ of the maximal dissipative operator $\widetilde{L}_{G}$ is a Blaschke product. The spectrum of $\widetilde{L}_{G}$ is purely discrete and belongs to the open upper half-plane. The operator $\widetilde{L}_{G}\left(G \neq G_{0}\right)$ has a countable number of isolated eigenvalues with finite multiplicity and limit points at infinity. The system of all eigenvectors and associated vectors of the operator $\widetilde{L}_{G}$ is complete in the space $H$.

Proof. From (3.1), it is clear that $S_{G}(\lambda)$ is an inner function in the upper half-plane, and it is meromorphic in the whole complex $\lambda$-plane. Therefore, it can be factored in the form

$$
S_{G}(\lambda)=e^{i \lambda c} B_{G}(\lambda), c=c(G) \geq 0,
$$

where $B_{G}(\lambda)$ is a Blaschke product. It follows from (4.1) that

$$
\left|S_{G}(\lambda)\right|=\left|e^{i \lambda c}\right|\left|B_{G}(\lambda)\right| \leq e^{-c(G) \operatorname{Im} \lambda}, \operatorname{Im} \lambda \geq 0 .
$$

Further, expressing $A_{G}(\lambda):=M(\lambda) K(\lambda)$ in terms of $S_{G}(\lambda)$, we find from (3.1) that

$$
A_{G}(\lambda)=\frac{\bar{G} S_{G}(\lambda)-G}{1-S_{G}(\lambda)} .
$$

For a given value $G(\operatorname{Im} G>0)$, if $c(G)>0$, then (4.2) implies that $\lim _{t \rightarrow+\infty} S_{G}(i t)=0$, and then (4.3) gives us that $\lim _{t \rightarrow+\infty} A_{G}(i t)=G_{0}$. Since $A_{G}(\lambda)$ does not depend on $G$, this implies that $c(G)$ can be nonzero at not more than a single point $G=G_{0}$ (and further $G_{0}=-\lim _{t \rightarrow+\infty} A_{G}(i t)$ ). This completes the proof.

\section{References}

[1] B.P. Allahverdiev, On dilation theory and spectral analysis of dissipative Schrödinger operators in Weyl's limit-circle case (Russian), Izv. Akad. Nauk. SSSR Ser. Mat.54 (1990), 242 - 257; English transl.: Math. USSR Izv. 36 (1991), $247-262$. 
[2] B.P. Allahverdiev, Spectral analysis of nonselfadjoint Schrödinger operators with a matrix potential, J. Math. Anal. Appl. 303 (2005), $208-219$.

[3] B.P. Allahverdiev, A nonself-adjoint singular Sturm-Liouville problem with a spectral parameter in the boundary condition, Math. Nachr. $278,7-8$ (2005), $743-755$.

[4] B.P. Allahverdiev, Spectral analysis of dissipative Dirac operators with general boundary conditions, J. Math. Anal. Appl. 283 (2003), $287-303$.

[5] B.P. Allahverdiev, Dilation and Functional Model of Dissipative Operator Generated by an Infinite Jacobi Matrix, Math. and Comp. Modelling 38,3 (2003), $989-1001$.

[6] M. Baro, H. Neidhardt Dissipative Schrödinger-type operators as a model for generation and recombination, J. Math. Phys. 44, 6 (2003), 2373 2401.

[7] M. Baro, H.-Chr. Kaiser, H. Neidhardt, J. Rehberg, Dissipative Schrödinger-Poisson systems, J. Math. Phys. 45, 1 (2004), 21 - 43.

[8] M. Baro, H.-Chr. Kaiser, H. Neidhardt, J. Rehberg, A Quantum Transmitting Schrödinger-Poisson System, Rev. Math. Phys. 16,3 (2004), $281-330$.

[9] J. Behrndt, M.M. Malamud, H. Neidhardt: Scattering theory for open quantum systems with finite rank coupling, Math. Phys. Anal. Geom. $10,(2007), 313-358$.

[10] J. Behrndt, M.M. Malamud, H. Neidhardt: Scattering matrices and Weyl functions, Proc. London Math. Soc. 97, (2008), 568 - 598.

[11] J. Behrndt, M.M. Malamud, H. Neidhardt: Trace formulae for dissipative and coupled scattering systems, Operator Theory Advances Applications $188,(2008), 49-87$.

[12] J. Behrndt, M.M. Malamud, H. Neidhardt: Finite rank perturbations, scattering matrices and inverse problems, Operator Theory Advances Applications 198, (2009), $61-85$.

[13] C. T. Fulton, The Bessel-squared equation in the lim-2, lim-3, and lim-4 cases, Quart. J. Math. Oxford (2), 40, (1989), 423- 456.

[14] M. L. Gorbachuk and V.I. Gorbachuk, Boundary Value Problems for Operator Differential Equations, Naukova Dumka, Kiev, 1984; English transl. 1991,Birkhauser Verlag. 
[15] A. N. Kochubei, Extensions of symmetric operators and symmetric binary relations, Mat. Zametki 17, (1975), 41 - 48; English transl. in Math. Notes 17 (1975), $25-28$.

[16] A. Kuzhel, Characteristic Functions and Models of Nonselfadjoint Operators, Kluwer Academic, Dordrecht, 1996.

[17] S. Kuzhel, On the inverse problem in the Lax-Phillips scattering theory method for a class of operator-differential equations, St. Petersburg Math. J. 13 (2002), $41-56$.

[18] P. D. Lax and R.S. Phillips, Scattering Theory, Academic Press, New York, 1967.

[19] B. Sz. Nagy and C., Foiaş, Analyse Harmonique des Operateurs de L'espace de Hilbert, Masson, Akad. Kiado, Paris, Budapest, 1967, English transl. North-Holland, Amsterdam, and Akad. Kiado, Budapest, 1970.

[20] M. A. Naimark, Linear Differential Operators, 2nd edn., 1968, Nauka, Moscow, English transl. of 1st. edn., 1, 2, 1969, New York.

[21] B. S. Pavlov, Selfadjoint Dilation of a Dissipative Schrödinger Operator and Eigenfunction Expansion, Funct. Anal. Appl., vol. 98, (1975), $172-$ 173.

[22] B. S. Pavlov, Selfadjoint Dilation of a Dissipative Schrödinger Operator and its Resolution in terms of Eigenfunctions, Math. USSR Sbornik, vol. 31, no.4, (1977), $457-478$.

[23] B. S. Pavlov, Dilation theory and spectral analysis of nonselfadjoint differential operators, Proc. 7th Winter School, Drobobych (1974), 3 69, (1976), (Russian); English transl: Transl. II. Ser., Am. Math. Soc. $115,(1981), 103-142$.

Hüseyin TUNA,

Department of Mathematics,

Mehmet Akif Ersoy University of Burdur,

15100 Burdur, Turkey.

Email: hustuna@gmail.com 